\title{
First in Village or Second in Rome?
}

\author{
EtTore Damiano \\ University of Toronto \\ Li, HaO \\ University of Toronto \\ WING SUEN \\ The University of Hong Kong
}

March 16, 2007

\begin{abstract}
Though individuals prefer to join groups with high quality peers, there are also advantages from being high up in the pecking order within a group. We show that sorting of agents in this environment results in an overlapping interval structure in the type space. Segregation and mixing coexist in a stable equilibrium. A greater degree of egalitarianism within organizations leads to greater segregation across organizations. Since competition is most intense for agents with intermediate talent, effective personnel policies to attract talent differ systematically between high-quality and low-quality organizations. When transfers are possible our stable equilibrium corresponds to a competitive equilibrium but entails too little segregation compared to the efficient assignment.
\end{abstract}

JEL Classification: C78, D83, M50

Corresponding Author: Ettore Damiano. Address: Department of Economics, University of Toronto, 150 St. George Street, Toronto, Ontario M5S3G7, Canada. Phone: (416) 946-5821. Fax: (416) 978-6713. E-mail: ettore.damiano@utoronto.ca.

Acknowledgments: We thank John Hartwick, Tony Latter, Aloysius Siow, Rich Romano, and Zhigang Tao for helpful comments, and Priscilla Man and Ron Chan for able research assistance. 
"I had rather be the first in this village than second in Rome."

-Attributed to Julius Caesar by Plutarch, Life of Caesar.

\section{Introduction}

Segregation and mixing typically coexist in the distribution of talents among organizations. For example, top ranked economists are exclusive to best research departments but at the same time second-tier departments often have economists who can easily make a successful career in any first-tier institution. A plausible explanation is that economists who prefer to have great colleagues also recognize that the more productive researchers within individual departments have greater access to departmental resources. This paper builds on this idea and develops an equilibrium sorting model to explain the pattern of talent distribution across organizations. The premise of our model is that interactions among individuals in an organization typically involve both cooperation and competition. In making mutually exclusive choices of which jobs to take, which schools to attend, or which social clubs to join, economic agents are concerned with both the "peer effect" and the "pecking order effect."

The peer effect is well-documented in the education literature (e.g., Coleman et al., 1966; Summers and Wolfe, 1977; Sacerdote 2001). Lazear (2001) has a model of educational technology in which the peer effect arises because learning is reduced for all other students when one student disrupts the class. More generally, the peer effect is seen as a consequence of the complementarity between characteristics of agents in a match. Naturally, people desire to join organizations with high-quality members if being in the company of highquality colleagues raises their own utility or productivity. But the motive to join the company of high-quality peers can also be present without complementarity. For example, potential employers' imperfect information about individual student quality leads to their use of the school average to improve on their estimate, as described in the statistical discrimination literature (e.g., Aigner and Cain, 1977). High-quality peers can therefore confer an informational externality on one another. 
The pecking order effect is related to people's concern for their relative status. It can arise from the effects on self-esteem developed through interactions with other agents in an organization. The idea that an individual's utility depends not only on the level of his consumption, but also on how that level compares with that of his reference group, has a long history in economics (e.g., Duesenberry, 1949; Frank, 1985; Solnick and Hemenway, 1998). In educational psychology, researchers speak of a "big fish-small pond" effect (Marsh and Parker, 1984). A study of academically talented students in Israeli primary schools finds that those who participate in special homogeneous classes for the gifted have lower academic self-concept and greater test anxiety than do those who participate in regular mixed-ability classes (Zeidner and Schleyer, 1998). ${ }^{1}$ The pecking order effect can also arise when some resources within a group or an organization are allocated through non-market means. Cole et al. (1992) describe a model in which the competition for mates leads to a concern for relative ranking. As Postlewaite (1998) points out, such kind of nonmarket allocation is ubiquitous. Resources within an organization such as corner offices or decision making power in recruitment are often allocated according to the relative status of the incumbents. Furthermore, people higher up in the pecking order have a greater chance of success in internal promotion tournaments. In school choices, the pecking order effect is present because grades are relative and depend on underlying abilities of fellow students. It is more important when grades have more serious implications, as demonstrated by the so-called top-ten-percent law in the state of Texas, which guarantees that students who finish in the top ten percent of their graduating class earn automatic admission to the Texas public university of their choice (The New York Times, April 14, 2002). ${ }^{2}$

Even men as great as Julius Caesar at some point might not be able to have it both ways. The rest of us are constantly reminded by the trade-off between the peer effect and

\footnotetext{
1 In a longitudinal study of secondary school students in Hong Kong, a city which has a highly achievement-segregated school system, Marsh et al. (2000) find that school-average ability has a negative effect on a student's academic self-concept, and that lower academic self-concept in turn adversely affects the student's subsequent academic achievement as reflected in standardized test scores. These researchers also find that higher perceived school status has a counterbalancing positive effect on self-concept. This is consistent with the informational externality we discuss in connection with the peer effect.
}

2 For empirical evidence that the adoption of the top-10-percent law in Texas influenced highschool students' enrollment decisions, see Cullen, Long and Reback (2005). 
the pecking order effect. Because the groups or organizations we are trying to join have limited capacities, individuals who are most desirable are expected to make any choice as they wish, while those who are least desirable have no real choice. It may thus appear that the sorting choices can be determined sequentially, with the more desirable agents choosing before the less desirable ones. But generally those ranked at the bottom of the first-tier organization will want to switch to the top of the second-tier organization. Sorting choices cannot be determined independently because for each agent both the peer effect and the pecking order effect in an organization depend on the choices made by other agents.

This paper presents a model where agents with heterogeneous, one-dimensional type face the trade-off between the peer effect and the pecking order effect in choosing between two organizations of fixed sizes. We model the peer efect by assuming that individual agents care about the average type in the organization they join, and we model the pecking order effect through the concern for their ranking according to type within the organization. While highly stylized, the model is meant to capture the essential features of sorting of talents in the presence of the peer and pecking order effects. We define a "sorting equilibrium" as an allocation of types among the organizations such that no agent whose type is higher than the lowest type in the other organization wishes to move. Sorting of agents is shown to result in an overlapping interval structure in the type space: the set of types that end up in each organization is an interval, and both the highest type and the lowest type are higher in the organization with a higher average type. Thus, in equilibrium segregation occurs for the top and the bottom types while the intermediate types mix between the two organizations. Further, the equilibrium overlapping-interval pattern of segregation and mixing is locally stable, so that small perturbations in agents' organization choices are self-correcting instead of self-fulfilling.

In our model the degree of segregation, measured in terms of differences in quality across organizations, increases as the concern for relative ranking becomes less important. ${ }^{3}$ In the context of school choice, this result suggests that a greater degree of egalitarianism

\footnotetext{
3 While it is known that the peer effect alone leads to complete segregation of agents by type (Becker, 1973; Benabou, 1993; Kremer, 1993), in our model complete segregation occurs in equilibrium if the pecking order effect is sufficiently weak, not necessarily absent.
} 
within schools can lead to greater segregation by ability across schools. ${ }^{4}$ The model also sheds light on how organizational policies affects the sorting of talents. In a sorting equilibrium with an overlapping interval structure, competition is most intense for agents with intermediate ability, because they are mobile across organizations. This suggests that a high-quality organization benefits from policies that cater more to its low-status members (who have intermediate ability) than to its high-status members, since the star agents have no better alternative than to stay. At the same time, the low-quality organization benefits from policies that cater more to its high-status members (who have intermediate ability) than to its low-status members, since the bottom agents have no real alternative but to stay.

Our characterization of equilibrium sorting and comparative statics analysis are based on a non-transferable utility model. In one interpretation of the model, complementarities exist between the agent type and the productivity of the relative position he holds in the organization. When transfers are allowed and agents bid competitively for positions of different ranking in the organizations, we show that our stable sorting equilibrium can be supported as a competitive equilibrium with price schedules for positions in different organizations. This is because the complementarity between the agent type and the relative position allows higher type agents to outbid lower types for higher-ranked positions in a competitive equilibrium. It turns out that this competitive equilibrium allocation does not maximize aggregate utility. When agents choose among organizations they do not internalize the peer effects on other agents, while the external effects on the pecking order average out across organizations. As a result, the competitive equilibrium allocation exhibits too little segregation. Under a linearity assumption on the form of complementarities between the agent type and the concerns for average quality and relative position, we show that maximizing aggregate utility requires sorting types into overlapping intervals as in the sorting equilibrium, and fully characterize the efficient allocation.

In the remainder of this section we review the existing literature that is most germane to the present study. In Section 2, we present the basic model where agents with identical

\footnotetext{
4 Similarly, for fixed salary structures, a lower team salary cap reduces the relative importance of the pecking order effect, and may result in a greater team quality difference, which is counter-productive in restoring the competitive balance of a sports league.
} 
trade-off between average quality and relative ranking sort into two ex ante identical, equalsized organizations. We show that all equilibria take the form of overlapping intervals. We define stable equilibrium and show that a unique stable equilibrium exists for any trade-off between average quality and relative ranking. In Section 3, we offer comparative statics results regarding system-wide and organization-specific factors that affect agents' concern for relative ranking. We also examine the effect on equilibrium sorting of uniform and idiosyncratic preference biases for organizations due to attributes independent of the quality-ranking trade-off. In Section 4 we introduce transfers and demonstrate that the sorting equilibrium we have identified can be implemented as a competitive equilibrium. Welfare analysis of the equilibrium shows that there is too little segregation in equilibrium compared to the efficient assignment. Section 5 concludes with a brief summary and a short discussion of some limitations of the present paper.

\subsection{Related literature}

There is a rich literature on the impact of the peer effect on sorting. The issue of streaming students by their ability has long been a controversial issue in education policy (e.g., Slavin 1987; Gamoran et al., 1995). One of the issues involved in this controversy is a conflict between efficiency and egalitarianism. Education researchers typically attribute the efficiency of ability streaming to the fact that teachers can tailor their instruction to a more homogeneous group of students (Lou et al., 2000). Economic theory suggests that positive assortative matching maximizes the sum of outputs if the production function is supermodular (Becker 1973). Lazear (1991) provides a micro-foundation for the existence of the peer effect in the classroom, and discusses the implications of the peer effect for streaming, class size, and other issues. Arnott and Rowse (1987) use a reduced-form education production function with peer effects to derive the optimal allocation of students and educational expenditure across classes. In the latter model, the extent of segregation by ability is limited by diminishing returns to educational expenditure.

In the literature on locational choices, De Bartolome (1990) develops a community choice model where families care about the provision of a public service (schooling) in the community. Families have high or low ability children. High ability children benefit more 
from expenditures on education (input effect) and all children benefit from having more high ability children in the school (peer effect). Segregation occurs with only input effect or peer effect, and when both effects are present mixed schools may obtain in equilibrium. The focus of that paper is on efficiency of school financing. Fernandez and Rogerson (1996) study a similar model but without the peer effect, and focus on welfare improving policies. They find that policies that increase the fraction of relatively wealthy individuals in the poorest neighborhood make everybody better off. In Epple and Romano (1998), students differ by ability and income and care about who their classmates are. Profit-maximizing private schools compete against each other and against a free public school by charging tuition according to the type of each student who enrolls. ${ }^{5}$ Their equilibrium has a segregated structure (i.e., no two schools enroll the same student type), and within each private school rich low-ability students subsidize poor high-ability ones. Becker and Murphy (2000, chap. 5) discuss the implications of the peer effect for residential segregation. They use a two-type model to illustrate how multiple equilibria, tipping, and inefficiency may arise in a competitive land market when people prefer to have "good" neighbors.

Kremer and Maskin (1996) study a one-sided matching model with a production function in which the more able worker within a two-person firm gets to perform the more productive task. ${ }^{6}$ Garicano and Rossi-Hansberg (2004) study how specialization and communication affect the equilibrium allocation of knowledge workers. By making the allocation of job positions endogenous, these two models capture some aspect of the pecking order effect. These models are particularly useful for analyzing earnings inequality, but they do not yield easily interpretable results concerning the degree of segregation by ability across organizations. In our paper, we use a reduced-form approach to study the pecking order effect, without committing to any specific channel in which relative ranks matter-be

\footnotetext{
5 Epple and Romano (1998) assume that the type of agents is observable. Damiano and Li (forthcoming) consider a random pairwise matching model with one-dimensional types and characterize price competition when type information is private.

6 Legros and Newman (2002) consider more general models and derive conditions for pairwise matching to be positive assortative. Hartwick and Kanemoto (1984) analyze the formation of a hierarchy of groups where each agent's utility from joining a group depends on a variable associated with the group and a value associated with the agent's rank within the group. There is no mixing in their equilibrium because the variable associated with the group depends only on a boundary type.
} 
it due to social psychological factors such as self-esteem, to non-market means of distributing resources within organizations, or to particular forms of production technologies. This approach allows us to develop a flexible model that yields unambiguous results concerning the systematic differences between high-quality and low-quality organizations.

\section{The Basic Model}

There is a mass of 2 of agents to be allocated into two organizations, $A$ and $B$. For simplicity, suppose that the organizations have the same capacity of 1 . Agents are characterized by their one-dimensional type $\theta$. The distribution of $\theta$ is given by the distribution function $F$ on the support $[\underline{\theta}, \bar{\theta}]$, with the corresponding density function $f$. We assume that $f$ is continuous.

Preferences of agents over the two organizations depend on the average type of agents in that organization, and on their individual ranking within that organization. ${ }^{7}$ For each $i=A, B$, let $m_{i}$ be the average type of agents in organization $i$. Let $r_{i}(\theta)$ be the quantile rank of an agent of type $\theta$ in organization $i$. Assume that the utility from joining organization $i$ is given by

$$
V_{i}(\theta)=\alpha r_{i}(\theta)+m_{i}
$$

where $\alpha$ is a positive weight that agents put on ranking relative to organization quality. ${ }^{8}$ The payoff is zero if an agent does not join either organization.

Equation (1) embeds three assumptions about the individual utility function. First, the concern for ranking and the concern for organization quality are additively separable;

7 Peer effects work through many channels, and we only consider the simplest formulation in this paper. If individuals prefer more homogeneous organizations, for example, then second moments would also enter into their preferences. One may also imagine more complicated models in which, say, the upper part of the distribution has a greater weight than the lower part because of the beneficial effects of role models.

8 We assume that preferences are continuous in rank. In many organizations, there may be a discontinuous payoff from being at the top. But since there is some uncertainty regarding which member of the organization will eventually end up being the top person, preferences will be continuous in the ex ante ability ranking. A similar remark also applies to the pecking order effect induced by the Texas top-ten-percent law. 
second, the marginal rate of substitution between relative rank and average quality is typeindependent; third, the marginal rate of substitution is constant. These assumptions allow us to characterize equilibrium pattern of sorting in a convenient way. We will comment on the role played by these assumptions in the analysis below. Some of our results apply to the more general case in which the marginal rate of substitution is type-independent but relative rank and average quality are not necessarily perfect substitutes. This case is represented by the individual utility function

$$
V_{i}(\theta)=v\left(r_{i}(\theta), m_{i}\right)
$$

where $v$ is increasing in both arguments and differentiable.

Equation (1) is the simplest functional form that satisfies the property that all agents face identical, constant trade-off between relative ranking and average quality. A more general form that retains this property allows interactions between types and the concerns for ranking and average quality, so that the utility function takes the multiplicatively separable form,

$$
V_{i}(\theta)=l(\theta)\left(\alpha r_{i}+m_{i}\right)
$$

for some positive-valued, strictly increasing, and differentiable function $l$. When there are no side transfers, equilibrium sorting is identical under equation (1) or (3); without loss of generality we carry out the analysis under equation (1). When utility is transferable, the form of $l$ matters. Later, we provide a more detailed discussion of equation (3) and use it to characterize competitive and efficient allocations.

\subsection{Overlapping intervals}

A feasible allocation in this model is a pair $\left(H_{A}, H_{B}\right)$ of cumulative type distributions in organizations $A$ and $B$ such that $H_{A}(\theta)+H_{B}(\theta)=F(\theta)$ for all $\theta \in[\underline{\theta}, \bar{\theta}]$. For each $i, j=A, B, i \neq j$, when $H_{i}(\theta)$ is constant in a neighborhood of some type $\theta$, types around $\theta$ are all allocated to organization $j$ only. If both $H_{A}$ and $H_{B}$ are strictly increasing in a neighborhood of $\theta$, then types around $\theta$ are split between $A$ and $B$. Let $T_{i}$ be the 
support set of organization $i$, defined as the closure of the set types at which $H_{i}$ is strictly increasing. In words, the support set $T_{i}$ is the set of types that do not exclusively choose organization $j$.

We assume that an agent can join organization $i$, either when the capacity of the organization is not yet filled, or when the capacity is filled but the agent's type is higher than the lowest type of that organization. Given this assumption, a natural equilibrium concept requires that at an equilibrium allocation no agent with the option of changing organization should strictly prefer to do so. Since the payoff from not joining an organization is zero, the capacity of each organization will always be filled. For the following definition, note that given a feasible allocation $\left(H_{A}, H_{B}\right)$, the rank $r_{i}(\theta)$ of type $\theta$ in each organization $i$ is well-defined, and is equal to $H_{i}(\theta)$.

Definition 1. A sorting equilibrium is a feasible allocation $\left(H_{A}, H_{B}\right)$ such that for each $i, j=A, B, i \neq j$, if $\theta \in T_{i}$ and $\theta>\inf T_{j}$, then $V_{i}(\theta) \geq V_{j}(\theta)$.

Our equilibrium concept is a natural notion of pairwise stability in a two-sided, manyto-one matching model with a continuum of agents on one side and two organizations on the other side. The preference of the agents is defined by equation (1). Each organization strictly prefers to add more agents of any type so long as the capacity constraint is not met, and for sets of agents that meet the capacity constraints, it prefers one with a higher average type to one with a lower average. ${ }^{9}$ Alternatively, Definition 1 corresponds to a subgame perfect equilibrium outcome of the following extensive form game between the agents and the two organizations. In the first stage of the game, all agents simultaneously choose to apply to either $A$ or $B$; in the second stage, after observing the pool of applicants each organization admits a subset of the pool of size at most equal to its capacity. In this game, the payoff of a type $\theta$ agent is given by equation (1) if admitted by organization $i$, and is zero if not admitted by either organization. The payoff to each organization is the

9 For an excellent analysis of two-sided matching models with finite agents, see Roth and Sotomayor (1990). Due to the payoff externalities introduced by the peer effect and the pecking order effect, the results surveyed by Roth and Sotomayor do not apply to our model. Further, in the model with finite agents, the payoff externalities cause difficulties in interpreting the notion of pairwise stability as an equilibrium concept. Such difficulties disappear in our model with a continuum of agents, because a deviation by a pair of an agent and an organization has a negligible impact on the payoffs of other agents. 
sum of the types of all admitted agents. It is straightforward to see that in any subgame perfect equilibrium outcome all agents are admitted by one of the two organizations and each organization has a size of 1 . The condition in Definition 1 then corresponds to the no deviation condition for type $\theta$ agent in a subgame perfect equilibrium.

Definition 1 is a simplification of reality. If agents have multi-dimensional attributes, it is not straightforward to define higher types and lower types. For example, an organization may hire workers who are less directly productive but who provide role models to improve its human capital stock (Athey et al., 2000). Even when agents can be unambiguously ranked on a single ability dimension, it is not always the case that incumbents in an organization necessarily welcome newcomers who have high ability. Our model is more likely to apply when entry into an organization is controlled by an outside decision maker who is primarily interested in maximizing the average quality of the organization. In Section 4 we allow side transfers and show that any equilibrium allocation according to Definition 1 can be supported as a competitive equilibrium where agents bid for positions of different ranking in organizations. Now we introduce a particularly simple form of allocation.

Definition 2. A feasible allocation is of an overlapping interval form if there exist types $x, y$, with $x \geq y$, such that the two support sets are $[y, \bar{\theta}]$ and $[\underline{\theta}, x]$.

In an overlapping interval form, the support set $T_{i}$ of each organization $i$ is an interval. There are two critical types $x$ and $y$, with $x \geq y$. All agents with type greater than $x$ go to one organization, say $A$. All agents with type lower than $y$ go to the other organization. Agents with type in the range $[y, x]$ are present in both organizations. The following lemma shows that all sorting equilibrium allocations take the form of overlapping intervals.

Lemma 1. Any sorting equilibrium allocation takes the overlapping interval form.

Proof. First we show that the support sets $T_{A}$ and $T_{B}$ are ordered in any sorting equilibrium: if $m_{A} \geq m_{B}$, then $\sup T_{A} \geq \sup T_{B}$ and $\inf T_{A} \geq \inf T_{B}$. Suppose $\sup T_{A}<$ $\sup T_{B}$. Then there is a type $\theta \in T_{B}$ such that $r_{B}(\theta)<1$ and $r_{A}(\theta)=1$. Since $m_{A} \geq m_{B}$, agents of type $\theta$ strictly prefer $A$. This is a contradiction since type $\theta$ has the option of moving to $A$. A similar argument establishes that inf $T_{A} \geq \inf T_{B}$. 
Next we show that in any sorting equilibrium the support set $T_{i}$ of each organization $i, i=A, B$, is an interval in the type space. Suppose not, and, without loss of generality, suppose that $T_{A}$ is not an interval. Then there exist two types $\theta$ and $\tilde{\theta}$, with $\theta<\tilde{\theta}$, such that all types on the interval $(\theta, \tilde{\theta})$ choose organization $B$ exclusively while types in small neighborhoods below $\theta$ and above $\tilde{\theta}$ are in the support set of organization $A$. Then, $H_{A}(\theta)=H_{A}(\tilde{\theta})$ and $H_{B}(\theta)<H_{B}(\tilde{\theta})$. Since types $\theta$ and $\tilde{\theta}$ have the option of switching between $A$ and $B$, both types must be indifferent between the two organizations, or

$$
\begin{aligned}
& \alpha r_{A}(\theta)+m_{A}=\alpha r_{B}(\theta)+m_{B} \\
& \alpha r_{A}(\tilde{\theta})+m_{A}=\alpha r_{B}(\tilde{\theta})+m_{B} .
\end{aligned}
$$

The above two contradict each other (recall that $r_{i}(t)=H_{i}(t)$ in each $i$.)

Lemma 1 contains two results: the support sets of the two organizations are ordered, and there are no gaps in the two support sets. The first result depends only on the assumption that the individual utility function $V_{i}(\theta)$ increases with $r_{i}(\theta)$ and $m_{i}$. The second result of no gaps in the support sets depends on the assumption that the marginal rate of substitution between relative ranking and average quality is type-independent in the individual utility function. However, even if relative ranking and average quality are imperfect substitutes as in equation (2), the contradiction argument for the no-gap result in the proof of Lemma 1 goes through. Thus, neither additive separability of the pecking order effect and the peer effect nor the constancy of the marginal rate of substitution is necessary for the result of overlapping intervals. ${ }^{10}$

While it has been recognized in the literature that positive complementarities lead to assortative matching and negative complementarities tend to produce mixing, there are few general results in the literature concerning the equilibrium allocation when both elements are present. In principle, "mixing" can take a variety of forms and this can render the

10 When the marginal rate of substitution depends on the type, there may be gaps in the support sets. For example, if the parameter $\alpha$ in equation (1) is replaced by a decreasing function of $\theta$, so that higher types are less concerned with the pecking order effect than lower types, then the support set of the higher quality organization may have gaps (the other support set continues to be an interval). 
analysis unwieldy. ${ }^{11}$ In our model, support sets contain no holes, and mixing takes the special form of overlapping intervals, drastically simplifying the subsequent analysis.

\subsection{Stable sorting equilibrium}

Fix an overlapping interval allocation. Without loss of generality, we assume that $T_{A}=$ $[y, \bar{\theta}]$ and $T_{B}=[\underline{\theta}, x]$. The restrictions on $H_{A}$ and $H_{B}$ imposed by the overlapping interval form are: (i) $H_{A}(\theta)=0$ and $H_{B}(\theta)=F(\theta)$ for $\theta \in[\underline{\theta}, y]$; (ii) $H_{A}(\theta)=F(\theta)-1$ and $H_{B}(\theta)=1$ for $\theta \in[x, \bar{\theta}]$; and (iii) $H_{A}(\theta)$ and $H_{B}(\theta)$ are strictly increasing for $\theta \in(y, x)$. See Figure 1 for an illustration. Note that feasibility of the allocation implies that $x \geq \theta_{e} \geq y$, where $\theta_{e}$ denotes the median type of agents in the population. There are two extreme cases of overlapping interval allocations: if $x=y=\theta_{e}$, we have a perfectly segregated allocation; if $x=\bar{\theta}$ and $y=\underline{\theta}$, and $H_{A}(\theta)=H_{B}(\theta)=\frac{1}{2} F(\theta)$, we have a perfectly mixed allocation.

If $x>y$ in an overlapping interval allocation, all agents of type $\theta \in[y, x]$ must be indifferent between organization $A$ and organization $B$. Recall that $r_{i}(\theta)=H_{i}(\theta)$ for each $i=A, B$. The indifference conditions for the threshold types $x$ and $y$ are

$$
\begin{aligned}
\alpha(2-F(x)) & =m_{A}-m_{B} ; \\
\alpha F(y) & =m_{A}-m_{B} .
\end{aligned}
$$

These two indifference conditions imply

$$
2-F(x)=F(y) \text {. }
$$

The above equation defines a one-to-one relation between $y$ and $x$, with $x=\bar{\theta}$ implying $y=\underline{\theta}$, and $x=\theta_{e}$ implying $y=\theta_{e}$. For all $\theta$ between $y$ and $x$, a similar indifference condition holds:

$$
\alpha\left(H_{B}(\theta)-H_{A}(\theta)\right)=m_{A}-m_{B} .
$$

11 In Kremer and Maskin (1996), workers with one-dimensional, heterogeneous types form pairwise matches to produce. There are two tasks, "manager" and "assistant," in each matched pair, and the production function is given by $\theta^{2} \tilde{\theta}$, where $\theta$ is the manager's type and $\tilde{\theta}$ is the assistant's type. When types are distributed uniformly over a relatively narrow range, total output is maximized by dividing the mass of workers into two equal-size overlapping intervals of types that are managers and of types that are assistants, and matching them positive assortatively. However, when the type distribution has a sufficiently large support, the set of types that are managers and the set of types that are assistants are no longer intervals. This feature makes the analysis of the extent of segregation in Kremer and Maskin (1996) quite intractable. 


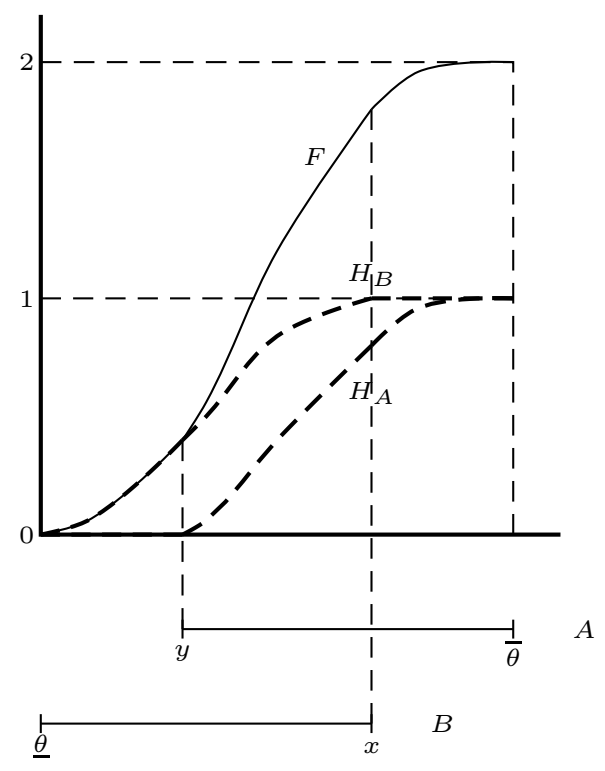

Figure 1

Thus, the difference in ranking for any type $\theta$ between $A$ and $B$ is constant on the interval $[y, x]$. This implies that types between $y$ and $x$ are evenly split between $A$ and $B$. Thus,

$$
H_{A}(\theta)= \begin{cases}0, & \text { if } \theta \leq y \\ \frac{1}{2}(F(\theta)-F(y)), & \text { if } y<\theta \leq x \\ F(\theta)-1, & \text { if } x<\theta \leq \bar{\theta}\end{cases}
$$

and $H_{B}(\theta)=F(\theta)-H_{A}(\theta)$.

With the above results, an overlapping interval allocation can be characterized by a single variable. It is more convenient to define an equilibrium in terms of the difference in average types, $m_{A}-m_{B}$. Denote $z=m_{A}-m_{B}$. To be consistent with our assumption that $T_{A}=[y, \bar{\theta}]$ and $T_{B}=[\underline{\theta}, x]$, we have $z \geq 0$. The largest possible value of $z$ is $\bar{\mu}_{e}-\underline{\mu}_{e}$, where $\bar{\mu}_{e}$ and $\underline{\mu}_{e}$ are the conditional mean above and below $\theta_{e}$ respectively. Given any $z \in\left[0, \bar{\mu}_{e}-\underline{\mu}_{e}\right]$, we use equations (4) to define the threshold types $x$ and $y$. Note that since $x \geq \theta_{e} \geq y$, equations (4) cannot be satisfied if $z>\alpha$ (this occurs only if $\alpha<\bar{\mu}_{e}-\underline{\mu}_{e}$ ); in this case we let $x=y=\theta_{e}$, as all types prefer $A$ to $B$ but only types above $\theta_{e}$ have the option of moving to $A$. Then, we can obtain $m_{A}$ as a function of $z$ :

$$
m_{A}(z)=\int_{\underline{\theta}}^{\bar{\theta}} t \mathrm{~d} H_{A}(t)
$$


where $H_{A}(t)$ is given by equation (7). From the identity $m_{A}+m_{B}=2 \mu$, where $\mu$ is the unconditional mean of the type distribution $F$, we get a necessary and sufficient condition for a sorting equilibrium with quality difference $z$ :

$$
D(z) \equiv 2\left(m_{A}(z)-\mu\right)=z .
$$

If there exists $z \in(0, \alpha)$ such that (8) is satisfied, then we have a sorting equilibrium with partial overlapping, where the threshold types $x$ and $y$ satisfy $\underline{\theta}<y<\theta_{e}<x<\bar{\theta}_{e}$ by equations (4). If equation (8) is satisfied by $z=\bar{\mu}_{e}-\underline{\mu}_{e}$, then we have a perfectly segregated equilibrium allocation, with $x=y=\theta_{e}$. Finally, since $z=0$ implies $x=\bar{\theta}$ and $y=\underline{\theta}$ by equations (4), and hence equation (8) is satisfied by $z=0$, a perfectly mixed allocation is always an equilibrium.

The equilibrium condition $D(z)=z$ may admit more than one solution in $z$ in the range $\left[0, \bar{\mu}_{e}-\underline{\mu}_{e}\right]$. However, some of these solutions may be "unstable" with respect to small perturbations in the equilibrium allocation. Following the standard stability concept, we say that a sorting equilibrium $z$ is stable if $D^{\prime}(z)<1$. $^{12}$ We have the following characterization result for the basic model.

Proposition 1. A unique stable sorting equilibrium exists for any $\alpha$, and is (a) perfect segregation if $\alpha \leq \bar{\mu}_{e}-\underline{\mu}_{e}$, (b) partial overlapping if $\bar{\mu}_{e}-\underline{\mu}_{e}<\alpha<\bar{\theta}-\underline{\theta}$, and (c) perfect mixing if $\alpha \geq \bar{\theta}-\underline{\theta}$.

Proof. We already know that $D(0)=0$ for any $\alpha$. Further, using equations (4) and (8), we can show that the derivative of $D(z)$ is given by

$$
D^{\prime}(z)=\frac{1}{\alpha}(x-y)
$$

From equations (4), we know that $x$ decreases and $y$ increases with $z$, and thus $D(z)$ is a concave function. There are three cases; see Figure 2 for an illustration. (a) If $\alpha \leq \bar{\mu}_{e}-\underline{\mu}_{e}$, then $D^{\prime}(0)>1$ and $x=y=\theta_{e}$ for all $z \in\left[\alpha, \bar{\mu}_{e}-\underline{\mu}_{e}\right]$. We have $D^{\prime}\left(\bar{\mu}_{e}-\underline{\mu}_{e}\right)=0$, and

12 We adopt the convention that $D^{\prime}(0)$ and $D^{\prime}\left(\bar{\mu}_{e}-\underline{\mu}_{e}\right)$ are defined by continuity, from above and from below respectively. Also, we say that $z=0$ is stable if $D^{\prime}(0)=1$ and $D(z)<z$ for $z$ just above 0 . 


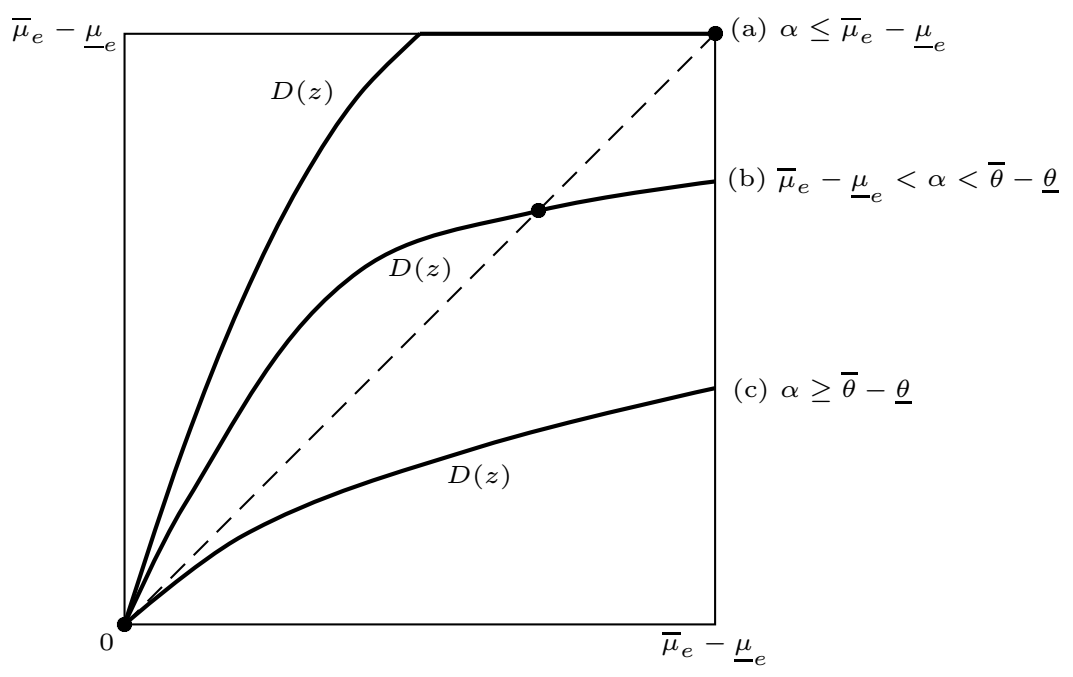

Figure 2

$z=\bar{\mu}_{e}-\underline{\mu}_{e}$ is the only stable solution. (b) If $\bar{\mu}_{e}-\underline{\mu}_{e}<\alpha<\bar{\theta}-\underline{\theta}$, then $D^{\prime}(0)>1$, and $D\left(\bar{\mu}_{e}-\underline{\mu}_{e}\right)<\bar{\mu}_{e}-\underline{\mu}_{e}$ because $x>\theta_{e}>y$ when $z=\bar{\mu}_{e}-\underline{\mu}_{e}$ from equation (4). There is a unique interior stable solution to $D(z)=z$. (c) If $\alpha \geq \bar{\theta}-\underline{\theta}$, then $D^{\prime}(0) \leq 1$ and therefore $D(z)<z$ for all $z>0$. The only solution to $D(z)=z$ is $z=0$, which is stable. Q.E.D.

The above proposition establishes the existence of a unique stable sorting equilibrium in our model for any utility parameter $\alpha$ and type distribution $F$. The uniqueness part of the result is due to the assumption of linear individual utility function (equation 1), but the existence part can be easily extended to the case when relative ranking and average quality are not perfect substitutes. As we have remarked about Lemma 1, when the individual utility function is instead given by equation (2), the support sets remain overlapping intervals. In general there will not be an even split of types that are allocated to both organizations, but we can follow the same procedure as above to construct a stable sorting equilibrium. ${ }^{13}$

13 For any $z$ in the permissible range of $\left[0, \bar{\mu}_{e}-\underline{\mu}_{e}\right]$, we can determine the threshold types $x$ and $y$ by $v\left(F(x)-1, m_{A}\right)=v\left(1, m_{B}\right)$ and $v\left(0, m_{A}\right)=v\left(F(y), m_{B}\right)$ respectively, while indifference conditions $v\left(H_{A}(\theta), m_{A}\right)=v\left(H_{B}(\theta), m_{B}\right)$ define the allocation $\left(H_{A}, H_{B}\right)$ between $y$ and $x$. A sorting equilibrium is then defined as before, as a fixed-point of equation (8). A stable equilibrium always exists: either $D^{\prime}(0) \leq 1$, in which case perfect mixing $(z=0)$ is a stable sorting equilibrium (because $\left.D(0)=0\right)$, or $D^{\prime}(0)>1$, in which case an interior stable equilibrium exists if $D\left(\bar{\mu}_{e}-\underline{\mu}_{e}\right)<\bar{\mu}_{e}-\underline{\mu}_{e}$ or otherwise perfect segregation $\left(z=\bar{\mu}_{e}-\underline{\mu}_{e}\right)$ is a stable equilibrium. 
Proposition 1 establishes that segregation and mixing generally coexist in equilibrium allocations of types across organizations. Since there is no inherent difference between organization $A$ and organization $B$, for any equilibrium in which $m_{A}>m_{B}$, there is another equilibrium involving the same allocation but with the identities of $A$ and $B$ reversed. Nevertheless, each equilibrium is locally stable in the sense that small disturbances to the allocation do not spread. In our model, "tipping" does not occur unless there is a large scale coordinated reallocation of individuals across organizations.

Since an agent of a higher type has more choices of organization than an agent of a lower type, equilibrium utility increases with type. In a perfectly mixed equilibrium allocation, the two organizations are identical and equilibrium utility increases at the rate of $\alpha f(\theta)$. In a perfectly segregated equilibrium, equilibrium utility increases twice as fast, at the rate of $2 \alpha f(\theta)$ in both organizations, with a discontinuity at $\theta=\theta_{e}$ since the median type strictly prefers $A$ to $B$. In a partially-overlapping allocation, equilibrium utility is continuous at the cutoffs $y$ and $x$, and increases at the rate of $2 \alpha f(\theta)$ for the segregating types in $[\underline{\theta}, y)$ and $(x, \bar{\theta}]$, and at the rate of $\alpha f(\theta)$ for the mixing types in $[y, x]$. Thus, for any fixed type distribution, equilibrium utility is more sensitive to type for segregated types. Inequality among agent types is more pronounced in a more segregated outcome.

\section{Comparative Statics Analysis}

The analysis leading to Proposition 1 shows that the equilibrium allocation can be reduced to a single variable $z$, which in turn depends on the parameter $\alpha$ and the distribution function $F$. As the equilibrium value of $z$ increases from 0 to $\bar{\mu}_{e}-\underline{\mu}_{e}$, the threshold type $x$ decreases from $\bar{\theta}$ to $\theta_{e}$ and $y$ increases from $\underline{\theta}$ to $\theta_{e}$. Thus, the equilibrium allocation becomes more segregated both in terms of a greater quality difference between the two organizations, and in terms of a smaller range of mixing. ${ }^{14}$

\footnotetext{
14 Kremer and Maskin (1996) define a segregation index as the ratio of between-organization variance to the sum of between-organization variance and within-organization variance. In our model, it can be shown the between-organization variance decreases with $x$, and the within-organization variance increases in $x$. Thus, the value of the Kremer-Maskin segregation index falls with $x$.
} 
In this section, we consider comparative statics regarding the degree of segregation. Part of this comparative statics analysis follows directly from the characterization of the unique stable sorting equilibrium in the previous section. In this case there is no ambiguity regarding how to measure the degree of segregation. In other cases we need to suitably extend the main model, by generalizing the overlapping interval form of allocation. ${ }^{15}$ Throughout this section we will identify the degree of segregation with the equilibrium average quality difference between the two organizations.

\subsection{Egalitarianism and segregation}

Suppose the pecking order effect increases, or the peer effect becomes less important. This change is represented by a rise in $\alpha$. Then, according to Proposition 1, perfect segregation becomes less likely to be the stable equilibrium while perfect mixing becomes more likely to be the stable equilibrium. Furthermore, in an equilibrium with a partial overlapping of support sets, equations (4) imply that the threshold type $x$ increases and $y$ decreases for any fixed $z$, with more types evenly split between $y$ and $x$. As a result, the whole function $D(z)$ shifts down as $\alpha$ increases. More precisely, we have

$$
\frac{\partial D(z)}{\partial \alpha}=-\frac{1}{\alpha}(x-y)(2-F(x))<0
$$

Thus the equilibrium $z$ decreases. We state this result as a proposition.

Proposition 2. An increase in the weight of the pecking order effect relative to the peer effect reduces the extent of segregation between two organizations.

The above result can be generalized to the case where relative ranking and average quality are not perfect substitutes in individual utility functions. As we have remarked on Proposition 1, stable sorting equilibria can be shown to exist when the individual utility function is given by equation (2). At any such equilibrium with allocation $\left(H_{A}, H_{B}\right)$ and

\footnotetext{
15 In an earlier version of the present paper, we also consider how the degree of segregation is affected when the relative size of the two organizations changes, when higher types care less about the pecking order effect, and when there are more than two organizations.
} 
quality difference $z$, when $\partial v\left(r_{i}, m_{i}\right) / \partial r$ increases at every $r_{i}$ and $m_{i}$, the equilibrium quality difference $z$ decreases. ${ }^{16}$

Proposition 2 has a number of applications. Suppose, for example, that a school board decides that all schools within its district adopt a more egalitarian approach to education. This means that top students within a school receive less attention and fewer scholarships or prizes, while students lower down the ladder of academic ability have greater access to the limited opportunities that help make a valuable educational experience (e.g., representing the school in external competitions). A more egalitarian policy can also be achieved by a more compressed distribution of grades, so that outsiders cannot so easily distinguish the top students from the bottom ones. Such a change in policy would lead to a fall in $\alpha$. As the advantages from being at the top of a school diminishes, agents will compete harder to enroll in the school with higher average student ability. Paradoxically, our analysis suggests that a policy toward greater egalitarianism within an organization may lead to an outcome with greater segregation across organizations.

In some professions, the peer effect comes from the fact that being associated with high quality colleagues tends to enhance one's human capital formation. To the extent that the pecking order effect arises from the consumption motive (e.g., self-esteem) while the peer effect arises from the investment motive, younger workers are expected to have a smaller relative weight $\alpha$ than do older workers. Proposition 2 then suggests that the distribution of talent across firms is more concentrated for younger cohorts of workers than it is for older cohorts.

So far we have only considered the effect of across-the-board changes in $\alpha$, but in reality organization policies are often not coordinated. Thus it is interesting to examine the effects

16 A brief argument is as follows. The indifference conditions $v\left(H_{A}(\theta), m_{A}\right)=v\left(H_{B}(\theta), m_{B}\right)$ in the overlapping interval can be rewritten as

$$
\int_{m_{B}}^{m_{A}} \frac{\partial v\left(H_{A}(\theta), m\right)}{\partial m} \mathrm{~d} m=\int_{H_{A}(\theta)}^{H_{B}(\theta)} \frac{\partial v\left(r, m_{B}\right)}{\partial r} \mathrm{~d} r .
$$

Note that $m_{A} \geq m_{B}$ and $H_{A}(\theta) \leq H_{B}(\theta)$. Thus, when $\partial v(r, m) / \partial r$ increases at every $r$ and $m$ (while $\partial v(r, m) / \partial m$ remains unchanged), organization $B$ becomes more attractive for each type $\theta$. Since $v$ is an increasing function of $r$, to restore the indifference condition $v\left(H_{A}(\theta), m_{A}\right)=v\left(H_{B}(\theta), m_{B}\right)$, we need to increase $H_{A}(\theta)$ and decrease $H_{B}(\theta)$. Similarly it can be shown that $x$ increases and $y$ decreases. As a result, $m_{A}(z)$ (and hence $D(z)$ ) decreases at each $z$, and the equilibrium value of $z$ decreases. 
of relative changes in $\alpha$. Consider an increase in $\alpha_{B}$, the weight on relative ranking in the lower quality organization, while $\alpha_{A}$ remains unchanged. This makes organization $B$ more attractive for all agents. In order to isolate the effect on equilibrium sorting arising from changes in $B$ that alter the trade-off between the pecking order effect and the peer effect in $B$, from the effect arising from changes that make $B$ universally more attractive, we look at a "compensated" change in $\alpha_{B}$. To do so, we assume that the utility for type $\theta$ from each organization $i=A, B$ is

$$
V_{i}(\theta)=\alpha_{i}\left(r_{i}(\theta)-\frac{1}{2}\right)+m_{i}
$$

Under this formulation, an increase in $\alpha_{B}$ means that all agents in organization $B$ ranked above the median become better off while those ranked below are made worse off. ${ }^{17}$ For example, a compensated increase in $\alpha_{B}$ occurs if organization $B$ adopts a more meritocratic personnel policy without increasing the overall level of compensation to its employees.

When $\alpha_{A} \neq \alpha_{B}$, we can still apply a similar argument as in Lemma 1 to show that the support sets of the two organizations are still intervals. However, in equilibrium one interval may strictly contain the other one. To see this, assume $\alpha_{A}>\alpha_{B}$ without loss of generality. The highest type, $\bar{\theta}$, prefers $A$ to $B$ if

$$
\frac{1}{2}\left(\alpha_{A}-\alpha_{B}\right)+z>0
$$

while the lowest type, $\underline{\theta}$, prefers $B$ to $A$ if

$$
-\frac{1}{2}\left(\alpha_{A}-\alpha_{B}\right)+z<0
$$

Thus, if in equilibrium the quality difference $z$ is between $-\frac{1}{2}\left(\alpha_{A}-\alpha_{B}\right)$ and $\frac{1}{2}\left(\alpha_{A}-\alpha_{B}\right)$, the very high types will all choose $A$, while the very low types are forced to stay with $A,{ }^{18}$ giving rise to a generalized form of overlapping intervals.

17 For simplicity we choose the median as the reference rank. From the following analysis we can see that the choice of the reference does not affect the mixing of types. Further, when $\alpha_{A}=\alpha_{B}$, the reference rank does not affect the range of mixing either. Thus the equilibrium under equation (9) is the same as the equilibrium under equation (1) when $\alpha_{A}=\alpha_{B}$.

18 In any sorting equilibrium agents of type $\underline{\theta}$ will never end up in $B$, which they strictly prefer. Otherwise, the lowest type in organization $A$ would strictly prefer to switch to $B$. See the proof of Proposition 3 in the appendix for a characterization of all sorting equilibria. 
We restrict our attention to comparative static analysis of the partially overlapping interval equilibria. Starting from one such equilibrium with $z>0$, when $\alpha_{B}$ increases, there is a stronger incentive for the threshold type $x$ to switch from $A$ to $B$. This tends to increase $x$, resulting in a decrease in $z$. We refer to this negative effect on the quality difference as the "threshold effect." When $\alpha_{B}$ increases, there is also an "allocation effect" on the quality difference, which is positive. To see this, note that for types $\theta \in[y, x]$ to be indifferent between the two organizations, we must have a constant difference $\alpha_{A} H_{A}(\theta)-\alpha_{B} H_{B}(\theta)$. This implies a mixing rule of a fraction $\alpha_{B} /\left(\alpha_{A}+\alpha_{B}\right)$ of each type $\theta$ going to $A$ and the rest going to $B$. Thus, an increase in $\alpha_{B}$ reduces the fraction of types in $[y, x]$ allocated to organization $B$. This tends to reduce $B$ 's average quality (for fixed thresholds $x$ and $y$ ). The following proposition provides a sufficient condition for the threshold effect to dominate the allocation effect, and therefore for an increase in $\alpha_{B}$ to narrow the degree of segregation between organizations $A$ and $B$.

Proposition 3. Suppose that the density function $f$ is symmetric about the median. At a partially-overlapping equilibrium with $z>0$ and $\alpha_{A} \geq \alpha_{B}$, a compensated increase in $\alpha_{A}$ lowers the equilibrium average quality $m_{A}$ of organization $A$, while a compensated increase in $\alpha_{B}$ raises the equilibrium average quality $m_{B}$ of organization $B$.

The proof of this proposition is in the appendix, where we also establish a sufficient condition on $\alpha_{A}$ and $\alpha_{B}$ for the existence of a partially-overlapping interval equilibrium. The intuition behind this result follows from the partially-overlapping structure. Recall that types below $y$ do not have the option of switching from organization $B$ to $A$. Reallocating resources away from these low types toward agents higher up in the hierarchy therefore makes organization $B$ more attractive to agents with relatively higher ability. Similarly, types above $x$ strictly prefer to stay in organization $A$. Reallocating resources away from these individuals toward agents lower down in the pecking order helps organization $A$ retain its talent. In a partially-overlapping allocation, since individuals with intermediate levels of ability are indifferent across organizations, competition for talent is most intense in this segment of the market. Thus, if the objective of organizations is to improve the overall quality of their incumbents, they should try to reallocate internal resources in order to attract this specific group of individuals. In a low quality organization, 
people with intermediate levels of ability are relatively high in the pecking order. So a more meritocratic approach to personnel policies that appeals to this group will enhance the quality of the organization. In a high-quality organization, however, meritocratic personnel policies are not appealing to people with intermediate levels of ability, since they are relatively low in the pecking order. Policies that are suitable for low quality organizations can be counterproductive for high quality organizations.

\subsection{Pre-existing differences in organizational attributes}

So far we have assumed that the quality of an organization depends solely on the average type of agents who choose it. But some organizations may be preferred for exogenous reasons, such as geographical location, physical endowments, and so on. Consider therefore a modification of the basic model in Section 2. Suppose that preferences for the low quality organization $B$ are instead given by

$$
V_{B}(\theta)=\alpha r_{B}(\theta)+m_{B}+\delta
$$

where $\delta$ is a parameter representing an attribute of organization $B$ that makes it more appealing relative to organization $A$. Modifying equations (4) and (6) with the addition of $\delta$, we can easily see that an increase in $\delta$ raises $x$ and reduces $y$, without affecting the result of an even split between $y$ and $x$. More precisely, we have

$$
\frac{\partial D(z)}{\partial \delta}=-\frac{1}{2 \alpha}(x-y)<0 .
$$

Thus, the whole function $D(z)$ shifts down, resulting in a smaller value of equilibrium $z$. This result is stated as the following proposition.

Proposition 4. An increase in the preference bias for the low quality organization reduces the extent of segregation between two organizations.

The comparative statics result remains valid when relative ranking and average quality are not perfect substitutes in individual utility functions, as in equation (2). ${ }^{19}$ Applying

19 With the preference bias $\delta$ for organization $B$, the equations that determine the threshold types become $v\left(F(x)-1, m_{A}\right)=v\left(1, m_{B}+\delta\right)$ and $v\left(0, m_{A}\right)=v\left(F(y), m_{B}+\delta\right)$. Since $v$ is an increasing function of $m$, an increase in $\delta$ raises $x$ and reduces $y$. Moreover, the indifference condition for a type $\theta \in(y, x)$ at a stable equilibrium with allocation $\left(H_{A}, H_{B}\right)$ becomes $v\left(H_{A}(\theta), m_{A}\right)=v\left(H_{B}(\theta), m_{B}+\delta\right)$. An increase in $\delta$ makes organization $B$ more attractive for $\theta$. To restore the indifference, we need to increase $H_{A}(\theta)$ and decrease $H_{B}(\theta)$. As a result, $D(z)$ decreases pointwise and the equilibrium value of $z$ decreases. 
our model to school choice, we can interpret $\delta$ as the tuition charged by the high quality school. ${ }^{20}$ Our result implies that a tuition increase by the high quality school reduces the extent of segregation by ability. Similarly, the parameter $\delta$ can be interpreted as the pay differential across organizations. For example, if wages are more compressed across law schools than they are across law firms, we would expect to see a greater degree of segregation by ability among law professors than among attorneys in the private sector.

Different agents may not value the other organizational attributes in the same way. For example, agents typically have different locational preferences. Let us consider a model where the utility of a type $\theta$ agent from choosing organization $B$ is given by

$$
V_{B}(\theta)=\alpha r_{B}(\theta)+m_{B}+\sigma \delta
$$

where $\sigma$ is a positive parameter representing the degree of heterogeneity in tastes, and $\delta$ is a random variable distributed according to $G$, with support $[\underline{\delta}, \bar{\delta}]$ and a continuous density function. For simplicity, we consider the case where the median of the distribution $G$ is zero, so that there is no aggregate bias for either organization.

Under equation (10), the equilibrium allocation takes the form of a generalized version of overlapping interval structures. When $\sigma$ is relatively large, then the highest type agents will be present in both organizations. In particular, if $\sigma \underline{\delta}<\underline{\mu}_{e}-\bar{\mu}_{e}$ and $\sigma \bar{\delta}>\bar{\mu}_{e}-\underline{\mu}_{e}$, the equilibrium allocation will not involve high type agents choosing exclusively the high quality organization. More generally, for each type $\theta$, the fraction of agents choosing the high quality organization $A$ increases in the quality difference $z$ and decreases in the ranking difference $r_{B}(\theta)-r_{A}(\theta)$. In the appendix, we show how to characterize the sorting equilibrium in this model, and establish the following result regarding the degree of heterogeneity in the idiosyncratic preferences for other organizational attributes.

Proposition 5. At a stable equilibrium, the degree of segregation decreases ( $z$ decreases) as the degree of heterogeneity in the idiosyncratic preferences increases ( $\sigma$ increases).

20 In Section 5 we consider the individual utility function (3) with side transfers and define competitive equilibrium in which agents bid for positions of different ranking in organizations. The price schedules in a competitive equilibrium are type-independent. The parameter $\delta$ introduced here (multiplied by the function $l$ ) may be interpreted as type-dependent tuition charges or wages. 
The logic of the above result is that a greater degree of heterogeneity in the idiosyncratic preferences reduces the importance of the trade-off between average quality and relative ranking to agents' organizational choices. For any quality difference $z$ there are more agents who prefer the low quality organization $B$ for idiosyncratic reasons as $\sigma$ increases. The equilibrium quality difference shrinks as a result.

The above result has an interesting implication for the design of benefit packages for organizations. Suppose an organization can choose between raising salaries by some total amount or providing in-kind benefits that cost the same amount. Suppose that different employees value the in-kind benefits differently. Then, our analysis suggests that the inkind benefits are more effective for the lower quality organization to attract talent, whereas cash wages are more effective for the higher quality organization to retain talent. Again, the intuition for this result follows from the overlapping interval allocation. Top talents in organization $A$ strictly prefer staying in $A$ to moving to $B$ under given cash wages. When the form of compensation switches to in-kind benefits, some of these top talents become better off because the benefits are worth more than the cash wages, but these individuals will stay in $A$ regardless. For those who value the in-kind benefits less than the cash wages, however, they will consider moving to organization $B$. On balance, therefore, increasing the variance of the value of benefits hurts the high-quality organization.

\section{Sorting with Transfers}

The utility function $V_{i}(\theta)=\alpha r_{i}+m_{i}$ is clearly inadequate for welfare analysis, because it would imply that aggregate utility is the same for any allocation. We therefore resort to the more general formulation of equation (3). Equation (3) may be justified by a model where in each firm workers need to be matched with tasks, and complementarity exists between the skill of a worker and the productivity of a task. Imagine that two firms, $A$ and $B$, need to each hire a unit mass of workers. In each firm, there is a continuum of tasks and each employed worker must be matched with a task. Tasks differ by the level of capital investment $k_{i}$ in each firm $i, i=A, B$. The productivity of task $k_{i}$ is $\alpha k_{i}+m_{i}$, 
where $m_{i}$ is the average skill level of workers in $i$. The output of a worker with skill level $\theta$ matched with task $k_{i}$ is given by

$$
l(\theta)\left(\alpha k_{i}+m_{i}\right)
$$

When the level of capital investment $k_{i}$ is uniformly distributed, we can assume without loss of generality $k_{i} \in[0,1]$ and identify a task with capital investment $k_{i}$ as a position of rank $k_{i}$. Then this production function (11) provides a rationale for the individual utility function in equation (3). We assume that the preference function of each agent is quasi-linear, given by the difference between the agent's utility (equation 3) and the agent's payment. As remarked earlier, unlike in previous analysis, $r_{i}$ in equation (3) is now a choice variable that ranges from 0 to 1 . To avoid confusion, we refer to $r_{i}$ as the level of a position, and use $H_{i}(\theta)$ as the quantile ranking of type $\theta$ in organization $i$. We call a feasible allocation of agents between the two organizations and a pairwise matching of agents with positions in each organization as an assignment.

\subsection{Competitive sorting}

We define a competitive equilibrium as an assignment together with two price schedules for positions, such that each agent chooses an organization and a position in the organization to maximize his utility, and markets clear with each agent getting exactly one position. Formally, let $p_{i}\left(r_{i}\right)$ be the price of position level $r_{i}$ in organization $i, i=A, B$. We have the following definition.

Definition 3. A competitive equilibrium assignment is a feasible allocation $\left(H_{A}, H_{B}\right)$ and price schedules $p_{A}$ and $p_{B}$, such that (a) if type $\theta$ is in the support set of $H_{i}, i=A, B$, then $i$ and $H_{i}(\theta)$ solves $\left.\max _{j=A, B}\left\{\max _{r_{j}} l(\theta)\left(\alpha r_{j}+m_{j}\right)-p_{j}\left(r_{j}\right)\right)\right\}$; and $(b) m_{i}=\int_{\underline{\theta}}^{\bar{\theta}} t d H_{i}(t)$.

The above definition implicitly assumes that in any competitive equilibrium assignment higher types are matched to higher positions in each organization. As a result, the market clearing condition is automatically satisfied. Note that since $l(\theta)$ is strictly increasing, the positive assortative matching of types to positions is implied by individual 
optimization and market clearing, because for any two positions $r_{i}>\tilde{r}_{i}$ in organization $i$, if a type weakly prefers $r_{i}$ to $\tilde{r}_{i}$ then any higher type strictly prefers $r_{i}$ to $\tilde{r}_{i}$.

We now argue that any sorting equilibrium allocation can be supported as a competitive equilibrium assignment. Suppose that $\left(H_{A}, H_{B}\right)$ is a sorting equilibrium, with threshold types $x$ and $y$. Without loss of generality, we assume that $m_{A} \geq m_{B}$. Then, the equilibrium ranking $H_{A}(\theta)$ of any type $\theta$ in $A$ satisfies equation (7), with the equilibrium ranking of $\theta$ in $B$ given by $H_{B}(\theta)=F(\theta)-H_{A}(\theta)$. The following proposition constructs price schedules and verifies that Definition 3 is satisfied. The reverse is also true: only sorting equilibrium allocations can be supported as competitive equilibrium assignments. This is established by first showing that in any competitive equilibrium assignment the two support sets $T_{A}$ and $T_{B}$ are intervals. Then, using the condition that types allocated to both organizations are indifferent, we show that these types must be evenly split between the two organizations. Since any competitive equilibrium assignment must satisfy the condition that the average type in each organization taken as given by each agent is precisely the average type that results from the organization choices of all agents (condition (b) in Definition 3), the support sets in the competitive equilibrium assignment must be overlapping intervals and the corresponding allocation is a sorting equilibrium allocation. The proof of the following proposition can be found in the appendix. ${ }^{21}$

Proposition 6. A feasible allocation can be supported as a competitive equilibrium assignment if and only if it is a sorting equilibrium.

The driving force behind Proposition 6 is the complementarity between the type of the agent and the relative position in the organization. When transfers are allowed and agents bid competitively for positions, this complementarity allows higher type agents to outbid lower types, and hence justifies our intuitive concept of sorting equilibrium when transfers are not allowed.

\footnotetext{
21 Proposition 6 holds so long as the marginal rate of substitution is type-independent, as in the case where the individual production function is given by $l(\theta) v\left(r_{i}, m_{i}\right)$.
} 


\subsection{Efficient sorting}

To understand the welfare properties of the equilibrium, let us consider a planner's problem of choosing an assignment of types to organizations and positions to maximize aggregate utility. We refer to the solution to the planner's problem as the efficient assignment. Since $l$ is an increasing function, given any allocation, the total utility of each organization is maximized by matching positions and agent types positive assortatively, as the average type $m_{i}$ is fixed, and complementarity exists between the type of agent and the level of position. Thus, for any feasible allocation $\left(H_{A}, H_{B}\right)$, type $\theta$ will be matched to position $r_{A}=H_{A}(\theta)$ in organization $A$ and the maximal total utility in $A$ is

$$
Q\left(H_{A}\right)=\alpha \int_{\underline{\theta}}^{\bar{\theta}} l(\theta) H_{A}(\theta) \mathrm{d} H_{A}(\theta)+\left(\int_{\underline{\theta}}^{\bar{\theta}} l(\theta) \mathrm{d} H_{A}(\theta)\right)\left(\int_{\underline{\theta}}^{\bar{\theta}} \theta \mathrm{d} H_{A}(\theta)\right) .
$$

Aggregate utility in the two organizations is $Q\left(H_{A}\right)+Q\left(H_{B}\right)$, where $H_{B}=F-H_{A}$.

The efficient assignment can be easily characterized for extreme values of $\alpha$. In particular, perfect segregation is efficient when $\alpha$ is sufficiently small, while perfect mixing is efficient for sufficiently large $\alpha \cdot{ }^{22}$ For intermediate values of $\alpha$ a characterization of the efficient assignment is difficult without any restriction on $l$. In the remainder of this subsection we assume that $l$ is linear, and equal to $\theta$ without loss of generality. In the appendix we prove the following necessary condition for efficient assignments. ${ }^{23}$

Lemma 2. Assume that $l$ is linear. Any efficient assignment takes the overlapping interval form with an even split of types allocated to both organizations.

\footnotetext{
22 To see this, when $\alpha$ is sufficiently small, the efficient assignment maximizes the second term in equation (12). The maximum is achieved when $m_{A}$ and $\int l(\theta) \mathrm{d} H_{A}(\theta)$ are made both as large as possible (or as small as possible), implying perfect segregation. When $\alpha$ is sufficiently large, the efficient assignment maximizes the first term in equation (12). Using integration by parts, we can show that the solution is $H_{A}(\theta)=H_{B}(\theta)$ for all $\theta$, implying perfect mixing.

23 If relative ranking and average quality are imperfect substitutes in individual utility function, types that are allocated to both organizations need not be evenly split in an efficient assignment. However, as long as the marginal rate of substitution between relative ranking and average quality is type-independent, as when the individual utility function is given by $\theta v\left(r_{i}, m_{i}\right)$, the support sets in any efficient assignment remain intervals. Thus, the no-gap result is a robust feature of efficiency, as is true in both sorting equilibrium and competitive equilibrium assignment.
} 
In the proof of Lemma 2, we use a local variation argument to show that mixing of types in the neighborhood of type $\theta$ implies that ${ }^{24}$

$$
\alpha\left(H_{A}(\theta)-H_{B}(\theta)\right)=2\left(m_{B}-m_{A}\right)
$$

Compare the above to equation (6). The next proposition follows immediately.

Proposition 7. There exists a unique efficient assignment for each $\alpha$, and it is identical to the sorting equilibrium corresponding to $2 \alpha$.

From Proposition 2, an immediate implication of Proposion 7 is that there is too little segregation in the sorting equilibrium. The inefficiency of the sorting equilibrium can be understood as follows. In a sorting equilibrium, agents choose organizations based on their individual utility, without any regard for the external effects of their decisions on the utility of other agents. Therefore, types that are allocated to both organizations in equilibrium must be indifferent (see equations 4 and 6). In particular, the equilibrium threshold $x$ is the highest type that weakly prefers the lower average type organization. In contrast, since there is more segregation at the efficient assignment than in the corresponding sorting equilibrium, we have

$$
\alpha(2-F(x))>m_{A}-m_{B}
$$

so that the threshold type $x$ strictly prefers $B$, the lower average type organization. The efficient assignment requires a lower threshold type $x$, and hence less mixing, because of the need to internalize the externalities. When the threshold $x$ is increased and some agents of types around $x$ are reallocated from the higher average type organization $A$ to $B$, it may appear that two kinds of externalities on other agents need to be internalized to maximize aggregate utility. One is that the agents with types between $y$ and $x$ now take on higher level positions in organization $A$ and lower level positions in organization $B$ (while those with types above $x$ and below $y$ keep their positions in the two organizations). This externality is not responsible for the discrepancy between the competitive assignment

\footnotetext{
24 This result relies on the linearity assumption on $l$. The general condition for any $\theta$ in a mixing interval is given by $\alpha\left(H_{B}(\theta)-H_{A}(\theta)\right)=\left(m_{A}-m_{B}\right)+\left(l_{A}-l_{B}\right) / l^{\prime}(\theta)$. Compare this to equation (13) below. The splitting rule for the efficient assignment is generally not even.
} 
and the efficient assignment, because the utility gain of each type in $A$ exactly cancels the utility loss of the same type in $B$. The other externality is that the average type in organization $A$ is decreased while the average type in $B$ is increased, making all agents in $A$ worse off and all agents in $B$ better off. This is the externality that fails to be internalized in a sorting equilibrium when agents with types around $x$ move from $A$ to $B$. Due to the complementarity between agent type and the average quality in an organization, agents of the threshold type $x$ must be discouraged from such a move to maximize aggregate welfare.

\section{Conclusion}

We have presented a sorting model with heterogeneous types where the equilibrium allocation of agents to organizations is determined by agents' concerns for average quality comparisons across organizations and for their relative ranking within the organizations. Sorting of agents is shown to result in an overlapping interval structure in the type space that allows coexistence of segregation and mixing. This result enables us to easily characterize the degree of segregation (measured by mean quality difference) across organizations. We show that a greater emphasis on relative ranking within organizations leads to less segregation across organizations. Moreover, because agents with intermediate ability are the most mobile across organizations, personnel policies that cater to this group (i.e., the low status members in high-quality organizations, and the high-status members in low-quality organizations) are particularly effective for raising the average quality of the organization.

The concept of sorting equilibrium introduced in this paper is appropriate when side payments are not possible so that sorting is primarily determined by the quality of agents. When transfers are possible, a more detailed specification of the production technology is necessary for analysis. We construct a model of endogenous task allocation with complementarity between agents and task as well as complementarity among agents in the same organization. In such an environment, the competitive equilibrium in which people bid for tasks in organizations corresponds precisely to our stable sorting equilibrium. The efficient sorting of agents to organizations in this environment also takes the form of an overlapping interval allocation, but efficiency requires a greater degree of segregation than 
in the sorting (or competitive) equilibrium. We caution, however, that our welfare results follow from our assumptions about production externalities. Alternative specifications of preferences and technologies that bring about the peer effect and the pecking order effect can lead to different welfare conclusions.

The main limitations of the present paper are the assumptions of capacity constraints and one-dimensional characteristics, and the absence of objective functions for organizations. Each of the three is worth further investigation. The assumption of capacity constraints may be profitably replaced by participation constraints on the side of agents or the side of organizations. The assumption of one-dimensional characteristics is standard in the sorting literature, but needs to be relaxed to enhance the applicability of our model. Finally, modeling the objective functions and strategies of organizations can further our understanding of equilibrium distribution of talents among organizations. Extension of the present model in these directions is beyond the scope of this paper, but the model presented here can prove fruitful in investigating these issues in sorting.

\section{Appendix}

Proof of Proposition 3. First, we provide a characterization of stable sorting equilibrium. Without loss of generality assume $\alpha_{A} \geq \alpha_{B}$. We allow $z$ to range from $\underline{\mu}_{e}-\bar{\mu}_{e}$ to $\bar{\mu}_{e}-\underline{\mu}_{e}$. For any such $z$, we can determine the support sets $T_{A}$ and $T_{B}$, as follows. Suppose for now $\frac{1}{2}\left(\alpha_{A}-\alpha_{B}\right)<\bar{\mu}_{e}-\underline{\mu}_{e}$. For any $z>\frac{1}{2}\left(\alpha_{A}-\alpha_{B}\right)$, we have $T_{A}=[y, \bar{\theta}]$ and $T_{B}=[\underline{\theta}, x]$, where $x$ and $y$ solve

$$
\begin{aligned}
\alpha_{A}\left((F(x)-1)-\frac{1}{2}\right)-\frac{1}{2} \alpha_{B}+z & =0 ; \\
-\frac{1}{2} \alpha_{A}-\alpha_{B}\left(F(y)-\frac{1}{2}\right)+z & =0 .
\end{aligned}
$$

For $z$ between $-\frac{1}{2}\left(\alpha_{A}-\alpha_{B}\right)$ and $\frac{1}{2}\left(\alpha_{A}-\alpha_{B}\right)$, we have $T_{A}=[\underline{\theta}, \bar{\theta}]$ and $T_{B}=[y, x]$, where $x$ and $y$ solve

$$
\begin{gathered}
\alpha_{A}\left((F(x)-1)-\frac{1}{2}\right)-\frac{1}{2} \alpha_{B}+z=0 \\
\alpha_{A}\left(F(y)-\frac{1}{2}\right)+\frac{1}{2} \alpha_{B}+z=0 . \\
-29-
\end{gathered}
$$


Finally, for $z<-\frac{1}{2}\left(\alpha_{A}-\alpha_{B}\right)$, we have $T_{A}=[\underline{\theta}, x]$ and $T_{B}=[y, \bar{\theta}]$, where $x$ and $y$ solve

$$
\begin{array}{r}
\frac{1}{2} \alpha_{A}-\alpha_{B}\left((F(x)-1)-\frac{1}{2}\right)+z=0 \\
\alpha_{A}\left(F(y)-\frac{1}{2}\right)+\frac{1}{2} \alpha_{B}+z=0 .
\end{array}
$$

If $\frac{1}{2}\left(\alpha_{A}-\alpha_{B}\right) \geq \bar{\mu}_{e}-\underline{\mu}_{e}$, then only the second case arises. In each of the three cases the resulting quality difference $D(z)$ is given by $2\left(m_{A}(z)-\mu\right)$, where $m_{A}(z)$ is the average quality in organization $A$ using the corresponding thresholds and the mixing rule of a fraction $\alpha_{B} /\left(\alpha_{A}+\alpha_{B}\right)$ of each type in $[y, x]$ going to $A$ and the rest going to $B$. As before, a stable sorting equilibrium is then a $z$ such that $D(z)=z$ and $D^{\prime}(z)<1$.

Next, we provide a sufficient condition for the existence of a partially-overlapping stable sorting equilibrium with $z>0$ when $\alpha_{A}>\alpha_{B}$. Start with $\alpha_{A}=\alpha_{B}=\alpha$. We argue that if $\alpha$ is between $2\left(\bar{\mu}_{e}-\mu\right)$ and $2(\bar{\theta}-\mu)$, then an overlapping equilibrium with $z>0$ exists when $\alpha_{A}$ marginally increases or $\alpha_{B}$ decreases marginally. To see this, note that $D(z)<z$ at $z=\bar{\mu}_{e}-\underline{\mu}_{e}$, because $\alpha_{A}, \alpha_{B}>2\left(\bar{\mu}_{e}-\mu\right)$ implies $x>\theta_{e}$ by equation (14). At $z=\frac{1}{2}\left(\alpha_{A}-\alpha_{B}\right)$, from equation (14) we have $F(\hat{x})=1+\alpha_{B} / \alpha_{A}$ and $F(\hat{y})=0$. Thus

$$
D\left(\frac{1}{2}\left(\alpha_{A}-\alpha_{B}\right)\right)=2\left(\mu-\int_{\underline{\theta}}^{\hat{x}} \frac{\theta f(\theta)}{F(x)} \mathrm{d} \theta\right) .
$$

By taking derivatives with respect to $\alpha_{A}$ or $\alpha_{B}$ at $\alpha_{A}=\alpha_{B}=\alpha$, we can show that $D\left(\frac{1}{2}\left(\alpha_{A}-\alpha_{B}\right)\right)>\frac{1}{2}\left(\alpha_{A}-\alpha_{B}\right)$ because $\alpha<2(\bar{\theta}-\mu)$. Thus there exists at least one stable equilibrium with partial overlapping.

Finally, for comparative statics when $\alpha_{A} \geq \alpha_{B}$, note that at any stable sorting equilibrium, the sign of $\mathrm{d} z / \mathrm{d} \alpha_{A}$ is the same as $\partial m_{A} / \partial \alpha_{A}$. We have

$$
m_{A}(z)=\int_{y}^{x} \frac{\alpha_{B}}{\alpha_{A}+\alpha_{B}} t f(t) \mathrm{d} t+\int_{x}^{\bar{\theta}} t f(t) \mathrm{d} t
$$

where $y$ and $x$ depend on $z$ and $\alpha_{A}$ through equation (14). The sign of $\partial m_{A} / \partial \alpha_{A}$ is the same as

$$
\left(F(x)-\frac{3}{2}\right) x+\frac{1}{2} y-\frac{\alpha_{B}}{\alpha_{A}+\alpha_{B}} \int_{y}^{x} t f(t) \mathrm{d} t .
$$


Upon integration by parts, and using equation (14), the above can be rearranged to

$$
\frac{x-y}{\alpha_{A}+\alpha_{B}}\left(F(x)-2+\frac{\alpha_{A}-\alpha_{B}}{2 \alpha_{A}}\right)+\frac{\alpha_{B}}{\alpha_{A}+\alpha_{B}} \int_{y}^{x}\left(F(t)-F\left(\theta_{e}\right)\right) \mathrm{d} t .
$$

We know that at $z=\frac{1}{2}\left(\alpha_{A}-\alpha_{B}\right)$, the threshold types satisfy $F(\hat{x})=1+\alpha_{B} / \alpha_{A}$ and $F(\hat{y})=0$. At any partially-overlapping interval equilibrium with $z>0$, we have $x<\hat{x}$, and hence the bracketed term in (15) is less than $F(\hat{x})-2+\frac{1}{2}\left(\alpha_{A}-\alpha_{B}\right) / \alpha_{A} \leq 0$. Furthermore, (14) and the symmetry of $f$ imply that $x-\theta_{e} \leq \theta_{e}-y$ when $\alpha_{A} \geq \alpha_{B}$. Therefore, the integral in (15) is also negative. We thus have $\mathrm{d} z / \mathrm{d} \alpha_{A}<0$ when $\alpha_{A}>\alpha_{B}$. Similar calculations show that $\mathrm{d} z / \mathrm{d} \alpha_{B}>0$ under the same conditions.

Proof of Proposition 5. Without loss of generality, assume $m_{A} \geq m_{B}$. The permissible range of $z$ is $\left[0, \bar{\mu}_{e}-\underline{\mu}_{e}\right]$. If $\sigma \underline{\delta}<\underline{\mu}_{e}-\bar{\mu}_{e}$ and $\sigma \bar{\delta}>\bar{\mu}_{e}-\underline{\mu}_{e}$, a positive fraction, $G(z / \sigma)$, of the highest type agents will choose $A$ and the rest will choose $B$. When $z>\sigma \bar{\delta}$ all agents of the highest type $\bar{\theta}$ will choose $A$.

Let $H_{A}(\theta)$ be the type distribution function in organization $A$. Then for any $\theta<\bar{\theta}$, the fraction of type $\theta$ agents that prefer $A$ to $B$ is given by $G\left(\left(z+\alpha\left(2 H_{A}(\theta)-F(\theta)\right)\right) / \sigma\right)$. Thus,

$$
H_{A}^{\prime}(\theta)=G\left(\frac{z}{\sigma}+\frac{\alpha}{\sigma}\left(2 H_{A}(\theta)-F(\theta)\right)\right) f(\theta)
$$

The above is a differential equation in $H_{A}$, with the boundary condition $H_{A}(\bar{\theta})=1$. (A unique solution to the differential equation (16) exists. See, for example, Verhulst (1996).) Let $H_{A}(\theta ; z)$ be the solution to the differential equation. We assume that the solution satisfies the capacity constraint of $A$; otherwise if the solution is negative for some $\theta$, redefine $H_{A}(\theta ; z)$ as 0 . (Note that the solution to the differential equation cannot exceed $F(\theta)$ at any $\theta$, hence the capacity constraint of $B$ is never violated.)

We have thus established that for any given $z$, there is a unique allocation of types between the two organizations consistent with the quality gap $z$. As before, an equilibrium with $z$ is defined by $D(z)=z$, and the equilibrium is stable if $D^{\prime}(z)<1$. Note that $D(0)=0$ because $H_{A}^{\prime}(\bar{\theta})=\frac{1}{2} f(\bar{\theta})$ and therefore the solution to the differential equation (16) is given by $H_{A}(\theta ; 0)=\frac{1}{2} F(\theta)$ for all $\theta$. At the other end, we have $D\left(\bar{\mu}_{e}-\underline{\mu}_{e}\right) \leq \bar{\mu}_{e}-\underline{\mu}_{e}$, 
with strict inequality if and only if $\sigma \bar{\delta}>\bar{\mu}_{e}-\underline{\mu}_{e}$. Thus, a stable sorting equilibrium always exists.

Now we prove the comparative statics result with respect to $\sigma$. We can show that an increase in $\sigma$ shifts $H_{A}(\theta ; z)$ downward in the sense that $H_{A}(\theta ; z)$ is ordered by first order stochastic dominance for any $z \in\left(0, \bar{\mu}_{e}-\underline{\mu}_{e}\right)$. To establish this claim, first note that at $\bar{\theta}$ the solution $H_{A}(\theta ; z)=1$ for all $\sigma$, and the slope is smaller for the solution corresponding to a greater $\sigma$. Next note that no two solutions corresponding to different $\sigma$ 's can cross at any other point, since at any crossing the solution corresponding to a greater $\sigma$ must have a smaller slope by equation (16). An increase in $\sigma$ then shifts down the function $D$. Since $D^{\prime}(z)<1$ at a stable equilibrium, the quality difference $z$ decreases.

Q.E.D.

Proof of Proposition 6. (i) Suppose that $\left(H_{A}, H_{B}\right)$ is a sorting equilibrium. For each organization $i=A, B$ and each position $r_{i} \in[0,1]$, let the price schedule $p_{i}$ be defined by

$$
p_{i}^{\prime}\left(r_{i}\right)=\alpha l\left(H_{i}^{-1}\left(r_{i}\right)\right)
$$

up to an integration constant to be determined below. Then, for any type $\theta \in T_{i}$, the first order condition of the optimization problem $\max _{r_{i}} l(\theta)\left(\alpha r_{i}+m_{i}\right)-p_{i}\left(r_{i}\right)$ is satisfied at $r_{i}=H_{i}(\theta)$. Since $p_{i}$ is convex by construction, the second order condition of the above optimization problem is satisfied. Thus, it is optimal for type $\theta \in T_{i}$ to choose the position of rank $H_{i}(\theta)$ among all positions in $i$. The implied indirect utility of $\theta$ is $U_{i}(\theta)=l(\theta)\left(\alpha H_{i}(\theta)+m_{i}\right)-p_{i}\left(H_{i}(\theta)\right)$, implying that

$$
U_{i}^{\prime}(\theta)=l^{\prime}(\theta)\left(\alpha H_{i}(\theta)+m_{i}\right)
$$

Given any $p_{A}(0)$ and $p_{B}(0)$, the indirect utility functions $U_{A}(\theta)$ (for $\theta \in[y, \bar{\theta}]$ ) and $U_{B}(\theta)$ (for $\theta \in[\underline{\theta}, x]$ ) are thus well-defined and continuously differentiable. Choose $p_{A}(0)$ and $p_{B}(0)$ such that $U_{A}(y)=U_{B}(y)$, and $U_{B}(\underline{\theta}) \geq 0$. Since $\left(H_{A}, H_{B}\right)$ is a sorting equilibrium, equation (6) is satisfied, and so it follows from equation (17) that $U_{A}(\theta)=U_{B}(\theta)$ for all $\theta \in[y, x]$.

It remains to argue that no type $\theta$ in $T_{i}$ but not $T_{j}$ will deviate from $i$ to $j$. Consider some type $\theta>x$ in $A$. The deviation utility $\tilde{U}_{B}(\theta)$ of this type in organization $B$ is 
maximized at $r_{B}=1$. This is because $\tilde{U}_{B}(\theta)$ is concave, and its derivative evaluated at $r_{B}=1$ has the same sign as $\alpha(l(\theta)-l(x))$, which is positive. To show that type $\theta$ will not deviate to $r_{B}=1$, we need

$$
U_{A}(\theta)>l(\theta)\left(\alpha+m_{B}\right)-p_{B}(1) .
$$

The above holds because by construction $U_{A}(x)=l(x)\left(\alpha+m_{B}\right)-p_{B}(1)$, and $U_{A}^{\prime}(\theta)=$ $l^{\prime}(\theta)\left(\alpha H_{A}(\theta)+m_{A}\right)$, which is strictly greater than $l^{\prime}(\theta)\left(\alpha+m_{B}\right)$. The argument for why it is not optimal for type $\theta<y$ to deviate from $B$ to $A$ is similar.

(ii) Let $\left(H_{A}, H_{B}\right)$ be a feasible allocation and suppose that $\left(H_{A}, H_{B}\right)$ can be supported by a competitive equilibrium assignment, with price schedules $p_{A}$ and $p_{B}$. Note that in any competitive equilibrium assignment each price schedule $p_{i}, i=A, B$, is continuous; otherwise, at a point of discontinuity, say $r_{i}$, there would be an excess supply for positions just above $r_{i}$. To show that the two support sets are both intervals, suppose without loss of generality that there is a gap in $T_{A}$, with types on the interval $[\theta, \tilde{\theta}]$ allocated to organization $B$ and at least some types just below $\theta$ and just above $\tilde{\theta}$ are allocated to $A$. Since $U_{B}(\theta)=U_{A}(\theta)$ by continuity, a necessary condition for the gap is $U_{B}^{\prime}(\theta) \geq \tilde{U}_{A}^{\prime}(\theta)$, where $U_{B}$ is the indirect utility for types between $\theta$ and $\tilde{\theta}$ and $\tilde{U}_{A}$ is the deviation utility for the same types. ${ }^{25}$ Since each type $t$ on the interval $(\theta, \tilde{\theta})$ must find it optimal to choose its equilibrium position $H_{B}(t)$ among all positions in $B$ ranked between $H_{B}(\theta)$ and $H_{B}(\tilde{\theta})$, the Envelope Theorem implies that $U_{B}^{\prime}(t)=l^{\prime}(t)\left(\alpha H_{B}(t)+m_{B}\right)$. Since a deviating type $t \in(\theta, \tilde{\theta})$ can always choose the position in $A$ corresponding to type $\theta$, we have $\tilde{U}_{A}^{\prime}(t) \geq l^{\prime}(t)\left(\alpha H_{A}(\theta)+m_{A}\right)$. Thus,

$$
l^{\prime}(\theta)\left(\alpha H_{B}(\theta)+m_{B}\right) \geq l^{\prime}(\theta)\left(\alpha H_{A}(\theta)+m_{A}\right) .
$$

Similarly, using the deviation condition of type $\tilde{\theta}$, we have

$$
l^{\prime}(\tilde{\theta})\left(\alpha H_{A}(\tilde{\theta})+m_{A}\right) \geq l^{\prime}(\tilde{\theta})\left(\alpha H_{B}(\tilde{\theta})+m_{B}\right) .
$$

25 Following the standard mechanism design literature (e.g. Stole, 1996), we can show that the indirect utility functions are differentiable almost everywhere. 
However, since $H_{A}(\theta)=H_{A}(\tilde{\theta})$ and $H_{B}(\tilde{\theta})>H_{B}(\theta)$, the above two inequalities contradict each other, implying that there cannot be a gap in $T_{A}$.

If the support sets of a competitive equilibrium assignment $\left(H_{A}, H_{B}\right)$ overlap on some interval $[y, x]$, then we have equation (17) from individual optimization of types on the interval within each organization $i$ among the positions ranked between $H_{i}(y)$ and $H_{i}(x)$. Since each such type $\theta$ is indifferent between $A$ and $B$, we have

$$
\alpha H_{A}(\theta)+m_{A}=\alpha H_{B}(\theta)+m_{B}
$$

The above is identical to the indifference conditions in a sorting equilibrium (see equation $6)$. Thus, types on the interval $[y, x]$ are evenly split between $A$ and $B$. It then follows from condition (b) in Definition 3 that $\left(H_{A}, H_{B}\right)$ is a sorting equilibrium.

Q.E.D.

Proof of Lemma 2. First, we show that if for some $\epsilon>0$ and some type $\theta, H_{B}$ is strictly increasing on $(\theta, \theta+\epsilon)$ and $H_{A}$ is strictly increasing on $(\theta-\epsilon, \theta)$, then

$$
\alpha\left(H_{A}(\theta)-H_{B}(\theta)\right) \leq 2\left(m_{B}-m_{A}\right)
$$

For any sufficiently small $\epsilon>0$, let $\gamma(\epsilon)$ solve

$$
H_{A}(\theta)-H_{A}(\theta-\gamma(\epsilon))=H_{B}(\theta+\epsilon)-H_{B}(\theta)
$$

Since $H_{B}$ is strictly increasing just above $\theta$ and $H_{A}$ is strictly increasing just below $\theta$, we have that $\lim _{\epsilon \rightarrow 0} \gamma(\epsilon)=0$. Define a new assignment $\left(H_{A}^{\epsilon}, H_{B}^{\epsilon}\right)$ such that

$$
H_{A}^{\epsilon}(t)= \begin{cases}H_{A}(t), & \text { if } t \notin(\theta-\gamma(\epsilon), \theta+\epsilon) \\ H_{A}(\theta-\gamma(\epsilon)), & \text { if } t \in(\theta-\gamma(\epsilon), \theta) \\ H_{A}(\theta-\gamma(\epsilon))+F(t)-F(\theta), & \text { if } t \in[\theta, \theta+\epsilon)\end{cases}
$$

and $H_{B}^{\epsilon}=F-H_{A}^{\epsilon}$. The assignment $\left(H_{A}^{\epsilon}, H_{B}^{\epsilon}\right)$ modifies $\left(H_{A}, H_{B}\right)$ by redistributing all agents of type on the interval $(\theta-\gamma(\epsilon), \theta)$ to organization $B$ and all types on the interval $[\theta, \theta+\epsilon)$ to organization $A$. Let $\Delta(\epsilon)$ be the difference in aggregate output between 
$\left(H_{A}^{\epsilon}, H_{B}^{\epsilon}\right)$ and $\left(H_{A}, H_{B}\right)$. We have

$$
\begin{aligned}
\Delta(\epsilon)= & \alpha\left(\int_{\theta}^{\theta+\epsilon} t\left(H_{A}(\theta-\gamma(\epsilon))+F(t)-F(\theta)\right) \mathrm{d} F(t)-\int_{\theta-\gamma(\epsilon)}^{\theta} t H_{A}(t) \mathrm{d} H_{A}(t)\right) \\
& +\alpha\left(\int_{\theta-\gamma(\epsilon)}^{\theta} t\left(F(t)-H_{A}(\theta-\gamma(\epsilon))\right) \mathrm{d} F(t)-\int_{\theta}^{\theta+\epsilon} t H_{B}(t) \mathrm{d} H_{B}(t)\right) \\
& +\left(m_{A}+\int_{\theta}^{\theta+\epsilon} t \mathrm{~d} H_{B}(t)-\int_{\theta-\gamma(\epsilon)}^{\theta} t \mathrm{~d} H_{A}(t)\right)^{2}-m_{A}^{2} \\
& +\left(m_{B}+\int_{\theta-\gamma(\epsilon)}^{\theta} t \mathrm{~d} H_{A}(t)-\int_{\theta}^{\theta+\epsilon} t \mathrm{~d} H_{B}(t)\right)^{2}-m_{B}^{2}
\end{aligned}
$$

Evaluating $\Delta(\epsilon)$ and its first two derivative at $\epsilon=0$ we have $\Delta(0)=\Delta^{\prime}(0)=0$ while $\Delta^{\prime \prime}(0)$ is proportional to $\alpha\left(H_{A}(\theta)-H_{B}(\theta)\right)-2\left(m_{B}-m_{A}\right)$. Thus, if $\alpha\left(H_{A}(\theta)-H_{B}(\theta)\right)>$ $2\left(m_{B}-m_{A}\right)$, it is possible to increase aggregate utility by redistributing agents of type close to $\theta$ according to $\left(H_{A}^{\epsilon}, H_{B}^{\epsilon}\right)$.

Next, we claim that the support set of each organization is an interval in the efficient assignment. To see this, suppose there is a gap in $T_{A}$. Then, there exist two types $\theta$ and $\tilde{\theta}$, with $\theta<\tilde{\theta}$, such that all types between $\theta$ and $\tilde{\theta}$ are allocated to $B$, and at least some types just below $\theta$ and just above $\tilde{\theta}$ are allocated to $A$. Then, Lemma 2 implies that

$$
\begin{aligned}
& \alpha\left(H_{B}(\theta)-H_{A}(\theta)\right) \geq 2\left(m_{A}-m_{B}\right) \\
& \alpha\left(H_{B}(\tilde{\theta})-H_{A}(\tilde{\theta})\right) \leq 2\left(m_{A}-m_{B}\right) .
\end{aligned}
$$

These two inequalities contradict each other, because $H_{A}(\theta)=H_{A}(\tilde{\theta})$ and $H_{B}(\theta)<H_{B}(\tilde{\theta})$. The lemma follows immediately, types that are allocated to both organizations must be evenly split, and a unit mass of agents must be allocated to each organization.

Q.E.D.

\section{References}

Aigner, Dennis J. and Glen G. Cain (1977), "Statistical Theories of Discrimination in the Labor Market," Industrial and Labor Relations Review 30, 175-187.

Arnott, Richard and John Rowse (1987), "Peer Group Effects and Educational Attainment," Journal of Public Economics 32, 287-305. 
Athey, Susan, Christopher Avery and Peter Zemsky (2000), "Mentoring and Diversity," American Economic Review 90, 765-786.

Benabou, Roland (1993), "Workings of a City: Location, Education, and Production," Quarterly Journal of Economics,108, 619-653.

De Bartolome, Charles A.M. (1990), "Equilibrium and Inefficiency in a Community Model with Peer Group Effect," The Journal of Political Economy 98, 110-133.

Becker, Gary S. (1973), "A Theory of Marriage: Part I," Journal of Political Economy 81, 813-846.

Becker, Gary S. and Kevin M. Murphy (2000), Social Economics, Cambridge, Harvard University Press.

Cole, Harold L., George J. Mailath and Andrew Postlewaite (1992), "Social Norms, Savings Behavior, and Growth," Journal of Political Economy 100, 1092-1125.

Coleman, J. S., E.Q. Campbell, C.J. Hobson, J. McPartland, A.M. Mood, F.D. Weinfeld and R.L. York (1966), Equality of Educational Opportunity, Washington, Government Printing Office.

Cullen, J. B., M.C. Long, and R. Reback (2005), "Jockeying for Position: High School Student Mobility and Texas' Top-Ten Percent Rule," Manuscript, UC San Diego.

Damiano, Ettore and Hao Li, "Competing Matchmaking," forthcoming Journal of the European Economic Association.

Duesenberry, James (1949), Income, Saving, and the Theory of Consumer Behavior, Cambridge, Harvard University Press.

Epple, Dennis and Richard E. Romano (1998), "Competition between Private and Public schools, Vouchers, and Peer-group Effects," American Economic Review 88, 33-62.

Fernandez, Raquel and Richard Rogerson (1996), "Income Distribution, Communities, and the Quality of Public Education," Quarterly Journal of Economics 111, 135-164.

Frank, Robert H. (1985), Choosing the Right Pond, Oxford, Oxford University Press.

Gamoran, A., M. Nystrand, M. Berends and P.C. LePore (1995), "A Organizational Analysis of the Effects of Ability Grouping," American Educational Research Journal 32, 687715 .

Garicano, Luis, and Rossi-Hansberg, Esteban (2004), "Inequality and the Organization of Knowledge," American Economic Review 94, 197-202.

Hartwick, John M. and Yoshitsugu Kanemoto (1984), "Formation of Convoys, Tennis Ladders, Colleges and Related Groups," Discussion Paper 589, Economics Department, Queen's University. 
Kremer, Michael (1993), "The O-Ring Theory of Economic Development," Quarterly Journal of Economics 108, 551-576.

Kremer, Michael and Eric Maskin (1996), "Wage Inequality and Segregation by Skill," NBER working paper 5718.

Lazear, Edward P. (2001), "Educational Production," Quarterly Journal of Economics $116,777-803$.

Legros, Patrick and Andrew F. Newman (2002), "Monotone Matching in Perfect and Imperfect Worlds," Review of Economic Studies 69, 925-942.

Lou, Y., P.C. Abrami and J.C. Spence (2000), "Effects of Within-Class Grouping on Student Achievement: An Exploratory Model," Journal of Educational Research 94, 101112 .

Marsh, Herbert W. and John W. Parker (1984), "Determinants of Student Self-Concept: Is It Better to Be a Relatively Large Fish in a Small Pond if You Don't Swim as Well?" Journal of Personality and Social Psychology 47, 213-231.

Marsh, Herbert W., Chit-Kwong Kong and Kit-Tai Hau (2000), "Longitudinal Multilevel Models of the Big-Fish-Little-Pond Effect on Academic Self-Concept: Counterbalancing Contrast and Reflected-Glory Effects in Hong Kong Schools," Journal of Personality and Social Psychology 78, 337-349.

The New York Times, "The 10 Percent Solution," Arpil 14, 2002.

Postlewaite, Andrew (1998), "The Social Basis of Interdependent Preferences," European Economic Review 42, 779-800.

Roth, Alvin and Marilda Sotomayor (1990), Two-sided Matching: A Study in Gametheoretic Modeling and Analysis, Cambridge University Press: Cambridge.

Sacerdote, Bruce (2001), "Peer Effects with Random Assignment: Results for Dartmouth Roommates," Quarterly Journal of Economics 116, 681-704.

Slavin, R.E. (1987), "Ability Grouping and Student Achievement in Elementary Schools: A Best-Evidence Synthesis," Review of Educational Research 57, 293-336.

Solnick, Sara and David Hemenway (1998), "Is More Always Better: A Survey on Positional Goods," Journal of Economic Behavior and Organization 37, 373-383.

Stole, L. (1996), "Lectures on the Theory of Contracts and Organizations," mimeo, University of Chicago.

Summers, A. and B. Wolfe (1977), "Do Schools Make a Difference?" American Economic Review 67, 639-652.

Verhulst, Ferdinand (1996), Nonlinear Differential Equations and Dynamic Systems, Berlin, 
Springer-Verlag.

Zeidner, Moshe and Esther Jane Schleyer (1998), "The Big-Fish-Little-Pond Effect for Academic Self-Concept, Test Anxiety, and School Grades in Gifted Children," Contemporary Educational Psychology 24, 305-329. 